\title{
Avaliação do modelo regional climático para a previsão de temperatura no centro de lançamento de Alcântara
}

\section{Evaluation of the climate regional model for temperature forecast at the Alcântara launch center}

DOI: $10.46814 / 1 a j d v 3 n 5-019$

Recebimento dos originais: 01/052021

Aceitação para publicação: 31/06/2021

\author{
Alexandre Santos de Souza \\ Instituto de Aeronáutica e Espaço/Divisão de Ciências Atmosféricas (IAE/ACA), São José dos \\ Campos, SP, Brasil. \\ Cleber Souza Correa \\ Instituto de Aeronáutica e Espaço/Divisão de Ciências Atmosféricas (IAE/ACA), São José dos \\ Campos, SP, Brasil. \\ Inácio Malmonge Martin \\ Instituto Tecnológico da Aeronáutica - Departamento de Física (ITA), São José dos Campos, SP, \\ Brasil.
}

\section{RESUMO}

Este estudo avaliou a previsão intrasazonal da temperatura à superfície ( $2 \mathrm{~m}$ de altura) na região do Centro de Lançamento de Alcântara (CLA) utilizando O Regional Climate Model RegCM4.7 em comparação aos dados de reanálises obtidos do modelo de reanalises globalERA5 do European Centre for Medium-Range Weather Forecasts (ECMWF) para os meses de abril de 2019 (estação chuvosa) e outubro de 2019 (estação seca). Foram realizadas 4 (quatro) membros de simulações de temperatura utilizando o RegCM4.7 em horários sinóticos, os quais foram comparadas com os dados observacionais e de reanálises do ERA5. Os resultados indicaram uma boa previsibilidade para a temperatura média nos dois períodos, diferenças inferiores a $1{ }^{\circ} \mathrm{C}$, com um grau de diferença para abril e praticamente coincidindo em outubro. Para as temperaturas máximas médias o RegCM4.7 superestimou em $2{ }^{\circ} \mathrm{C}$ para abril e $4{ }^{\circ} \mathrm{C}$ para outubro. Para as temperaturas médias mínimas subestimou em $2{ }^{\circ} \mathrm{C}$ tanto para abril como para outubro. Essas avaliações indicaram um bom desempenho geral para a previsão de temperaturas médias, contudo, sabendo-se da tendência de superestimar temperaturas máximas médias e subestimar temperaturas mínimas médias, ainda assim, com as devidas correções, poderá ser utilizado com eficácia para a previsão intrasazonal de temperatura à superfície em apoio ao planejamento de operações de lançamento no CLA.

Palavras-chave: Modelo Climático Regional, Previsão Intrasazonal, Operações de Lançamento de Foguetes, Centro de Lançamento de Alcântara.

\begin{abstract}
This study evaluated the intraseasonal surface temperature $(2 \mathrm{~m}$ height $)$ forecast in the Alcântara Launch Center (ALC) region using the Regional Climate Model RegCM4.7 against reanalysis data obtained from the European Center for Medium-Range Weather global Forecasts (ECMWF) reanalysis model ERA5 for the months of April 2019 (rainy season) and October 2019 (dry season). Four members of temperature simulations were performed using RegCM4.7 at synoptic times, which were compared with observational and reanalysis data from ERA5. The results indicated a good
\end{abstract}


predictability for the average temperature in the two periods, differences below $1{ }^{\circ} \mathrm{C}$, with one degree of difference for April and practically coinciding in October. For average maximum temperatures RegCM 4.7 overestimated by $2{ }^{\circ} \mathrm{C}$ for April and $4{ }^{\circ} \mathrm{C}$ for October. For average minimum temperatures, it was underestimated by $2{ }^{\circ} \mathrm{C}$ for both April and October. These evaluations indicated a good overall performance for predicting average temperatures, however, knowing the tendency to overestimate average maximum temperatures and underestimate average minimum temperatures, even so, with the appropriate corrections, it can be used effectively for forecasting intraseasonal surface temperature analysis in support of ALC launch operations planning.

Keywords: Regional Climate Model, Intraseasonal Forecasting, Rocket Launch Operations, Alcântara Launch Center.

\section{INTRODUÇÃO}

A principal fonte de energia do nosso planeta é recebida do Sol, e a principal contribuição para o balanço de energia da Terra é a Irradiação Solar Total que possui influência grande nos sistemas meteorológicos, que em geral, são bem conhecidos e possuem boa previsibilidade em períodos curtos ( $<7$ dias), já para previsões de médio e longo prazo, existem grandes desafios para melhorias na previsibilidade [1]. Muitos esforços estão sendo feitos e foram alcançados avanços significativos nos últimos anos, principalmente na previsão intrasazonal. Este estudo preliminar pretende avaliar a previsão intrasazonal de temperatura obtidas com o modelo climático RegCM4.7 para a Região do CLA. Uma estimativa da temperatura média à superfície com alguns meses de antecedência irá contribuir para um melhor planejamento das operações de lançamento, a temperatura à superfície (2 m de altura) é um parâmetro operacional que afeta o lançamento de foguetes.

O sucesso das atividades espaciais depende de muitos fatores, dentre eles, destaca-se o conhecimento da meteorologia da região do centro de lançamento, para tal estudos climatológicos são muito importantes e apoiarão as tomadas de decisões desde as fases de planejamento, transporte de equipamentos até a operação de lançamento [2].

Para o devido apoio ao planejamento das operações no CLA, são utilizadas informações climatológicas e previsões climáticas. Diante do desafio de melhorar a previsibilidade é necessário o acompanhamento constante dos avanços científicos e tecnológicos e buscando melhorias nas previsões climáticas.

Nesse contexto, o Modelo Climático Regional (Regional ClimateModel - RegCM4.7), é uma importante ferramenta a ser utilizada para a previsão intrasazonal, pois utiliza metodologia atualizada, baseada na técnica de redução de escala, oferecendo previsões climáticas mais detalhadas em comparação com os Modelos Climáticos Globais (Global Climate Models - GCMs) e poderá ser 
utilizado operacionalmente para um melhor apoio ao planejamento e assim proporcionar maior segurança e economia às operações de lançamento de veículos aeroespaciais a partir do CLA [3].

Antes de falar do RegCM, um dos diversos Modelos Climáticos Regionais (Regional ClimateModesl - RCMs), é necessário explanar um pouco sobre os Modelos Climáticos Globais (Global ClimateModels- GCMs), os quais são amplamente utilizados para previsões de grande escala de espaço e tempo, sua resolução horizontal é da ordem de 200 km [4] e suas previsões vão de meses até anos ou décadas, é necessário um grande desempenho computacional e de alto custo, visto que seus cálculos representam as características climáticas em larga escala para todo o planeta, como a circulação geral da atmosfera e dos oceanos [5].

Contudo, apesar desses modelos serem úteis para uma visão geral do clima, sua grade de baixa resolução não traz uma visão detalhada regionalmente, o que para atividades específicas como lançamento de foguetes seria extremamente importante.

Os RCMs, necessitam de inicialização a partir de GCMs, pois "modelos climáticos regionais utilizam condições iniciais e de contorno oriundas de GCMs" [4], mas os RCMs proporcionam grande vantagem em sua utilização, possuindo uma grade horizontal menor, da ordem de $50 \mathrm{~km}$, apresentando maior resolução espacial. Sendo assim, adicionam muito mais detalhes às previsões, além de demonstrarem um custo computacional muito menor em comparação com os GCMs.

O RegCM apresenta uma metodologia atualizada com a técnica de redução de escala, conhecida como "downscaling", a qual seu objetivo é obter detalhes regionais ou locais a partir de observações esparsas ou simulações numéricas de baixa resolução [5]. Suas simulações numéricas baseadas em características dinâmicas da atmosfera apresentam diferencial positivo quando comparadas com a climatologia e com os GCMs que possuem grade espacial de menor resolução e menos informações de cobertura vegetal e relevo.

Dessa forma o RegCM proporciona previsões climáticas mais detalhadas, como afirma [5] "Modelos climáticos regionais adicionam mais detalhes às simulações globais do clima, permitindo estudo dos processos climáticos com mais detalhes do que os modelos globais permitem".

Os RCMs tiveram o início de sua utilização no final dos anos 80 , com a proposta de melhoria dos resultados das simulações de GCMs. Antes, porém, é importante entender sua origem e estado da arte, verificando os avanços científicos dos últimos 30 anos, suas características e limitações. O pioneiro RCM foi o RegCM, desenvolvido na National Center for Atmospreric Research (NCAR) pelos pesquisadores [6], [7], e [8], com a proposta de aninhamento de alta resolução em saídas de simulações climáticas de GCMs [9]. Atualmente o RegCM é mantido pelo Abdus Salam International Center for Theoretical Physics (ICTP). 
Nesse sentido, o RegCM tem apresentado bom desempenho nas simulações climáticas sazonais na América do Sul como afirmam os estudos utilizando o RegCM3 que avaliou os efeitos de parametrizações na simulação da estação chuvosa na América do Sul [10], a versão RegCM4.3 foi submetida à análises de sensibilidade para esquemas de parametrização física [11], e para simulações climáticas sazonais no Brasil, a versão RegCM4.5 [12]. Para a Região Nordeste do Brasil [13] fez uma análise mostrando o grande potencial do uso da previsão ensemble para previsão de intensidade do vento no Centro de Lançamento de Alcântara utilizando o RegCM4.6. Também para o CLA [3] fez um estudo para estimativas de vento médio utilizando o RegCM4 inicializados com 3 diferentes condições globais iniciais.

Dessa forma, vemos a grande importância que o RegCM tem tido nos últimos anos, e será de muita utilidade as previsões intrasazonais deste modelo para o apoio ao planejamento das operações de lançamento.

Uma maneira de poder avaliar o desempenho do modelo é comparar as simulações com dados observados, o parâmetro de temperatura é bastante interessante para ser avaliado, inicialmente, por ser um dado contínuo, facilita a análise dos dados e principalmente por ser de grande utilidade nos preparativos para os lançamentos, pois os componentes eletrônicos dos foguetes devem estar sob um monitoramento constante dentro do range de capacidade de operação, além também da temperatura do ar ser utilizada para os cálculos de refração atmosférica que auxiliam no rastreio dos foguetes após lançamento.

Este trabalho tem o objetivo de avaliar o modelo RegCM4.7 na previsão intrasazonal de temperatura na superfície na região do CLA para os meses de abril e outubro de 2019. Para tal veremos nos próximos capítulos, a metodologia utilizada indicando a forma de avaliação do modelo através das análises das comparações das simulações com os dados observacionais, seguido a análise dos resultados com a discussão da previsão de temperatura para a região do CLA verificando-se a acurácia do modelo e diferenças entre a previsão de temperatura num mês considerado chuvoso (abril) e num outro mês considerado seco (outubro), conforme climatologia local [14]. Por fim as considerações finais indicando a utilização do RegCM4.7 como importante ferramenta para a previsão intrasazonal de temperatura na superfície na região do CLA em estações sazonais diferentes como os meses de abril de 2019 e outubro de 2019.

\section{METODOLOGIA}

Para a previsão intrasazonal na região do CLA, utilizou-se do modelo RegCM4.7 inicializado com o modelo (global Coupled Forecast System modelversion 2 NCEP CFSv2). O RegCM4.7 foi obtido no site do International Center for Theoretical Phisics (ICTP) [15], foram feitas as instalações e 
ajustes dos parâmetros físicos conforme trabalho citado anteriormente [3]. Da mesma forma os dados de inicialização do Modelo Global CFSv2 foram obtidos do site do National Centers for Enviromental Prediction (NCEP) [16].

$\mathrm{O}$ parâmetro estudado foi a temperatura na superfície para a região entre as latitudes $01^{\circ} \mathrm{N} \mathrm{e}$ $06^{\circ} \mathrm{S}$ e entre as longitudes $041^{\circ} \mathrm{W}$ e $048^{\circ} \mathrm{W}$, nos meses de abril de 2019 e outubro de 2019 para a verificação da qualidade da previsão de temperatura na superfície na região do CLA em estação chuvosa e seca, respectivamente. Foram realizadas 4 simulações para cada mês de interesse, sendo a primeira iniciada quatro meses antes (jan-abr/jul-out), a segunda três meses antes (fev-abr/ago-out), a terceira um mês antes (mar-abr/set-out) e a quarta simulação no mês de interesse (abr-abr/out-out).

Para verificar a eficiência do modelo, foram obtidos dados de reanálise do European Centre for Medium-Range WeatherForecasts ECMWF [17] para a mesma região de estudo nos meses de abril de 2019 e outubro de 2019.

A idéia foi de comparar as simulações do RegCM4.7 com os dados de reanálise ERA5 para a região do CLA. Para tal, foi necessário um pré-processamento para ajuste dos dados, através do software Climate Data Operator (CDO) [18] os dados foram normalizados para uma mesma grade de $02.5^{\circ}$ de resolução e sincronizados no tempo, em horários sinóticos a cada três horas a partir das $00 \mathrm{~h}$ de Tempo Universal Coordenado (Universal Time Coordinated - UTC), para viabilizar a devida comparação. Também foi utilizado o CDO para cálculos estatísticos e para obtenção dos dados no ponto de coordenadas do CLA $\left(02^{\circ}, 15^{\prime} \mathrm{S}\right.$ e $\left.044^{\circ}, 30^{\prime} \mathrm{W}\right)$, também foi utilizado o software Grid Analysisand Display Sistem(GrADS) [19] para visualização do campo de temperatura para uma região mais abrangente em torno do CLA (latitudes de $01^{\circ} \mathrm{N}$ a $06^{\circ} \mathrm{S}$ e longitude de $041^{\circ} \mathrm{W}$ a $048^{\circ} \mathrm{W}$ ).

Com os campos de temperatura previstos e observados foram obtidas figuras para comparação visual, e para obter maiores detalhes, foram construídas matrizes de dados de temperatura na superfície para os dados simulados do RegCM4.7 e para os dados observados do ERA5 para a coordenada do CLA $\left(02^{\circ}, 15^{\prime} \mathrm{S}\right.$ e $\left.044^{\circ}, 30^{\prime} \mathrm{W}\right)$. Foram feitas as diferenças entre os valores previstos e observados das temperaturas máximas médias, médias e mínimas médias para os meses de estudo. Verificando-se a qualidade da previsão do RegCM4.7 nas simulações de temperatura na superfície para a Região do CLA.

\section{ANÁLISE DOS RESULTADOS:}

Os resultados obtidos foram expressos nos gráficos a seguir, onde primeiramente visualizamse as comparações entre as reanálises e simulações para os meses de abril de 2019 e outubro de 2019. Inicialmente foram geradas as figuras de temperaturas médias mensais do ERA5 e RegCM4.7 para uma análise subjetiva em torno da área do CLA. 
Nessa análise verifica-se que, tanto para o mês de abril de 2019 como para outubro de 2019, as temperaturas médias previstas e observadas estiveram bem próximas na região do CLA $\left(02^{\circ}, 15^{\prime} \mathrm{S}\right.$ e $044^{\circ}, 30^{\prime} \mathrm{W}$ ), com pequenas diferenças em torno de $1{ }^{\circ} \mathrm{C}$ a $2{ }^{\circ} \mathrm{C}$, mas para regiões mais ao Sul, dentro do continente, nas simulações, percebem-se tendências de subestimar as temperaturas médias em torno de $4{ }^{\circ} \mathrm{C}$ em abril de 2019 e superestimar as temperaturas médias em até $5{ }^{\circ} \mathrm{C}$ em outubro de 2019 , conforme Figuras 1 e 2 .

Após essa análise inicial mais subjetiva, buscou-se uma análise mais aprofundada e objetiva, para a região do CLA $\left(02^{\circ}, 15^{\prime} \mathrm{S}\right.$ e $\left.044^{\circ}, 30^{\prime} \mathrm{W}\right)$, realizando a comparação entre as temperaturas mensais máximas médias, médias e mínimas médias simuladas e observadas nos horários sinóticos para verificação da destreza do modelo. Verificou-se que o modelo acompanha bem o ciclo diário de temperatura e que na comparação entre os meses de estudo, abril apresentou menor diferença entre as simulações e observações, com diferença máxima em torno de $2^{\circ} \mathrm{C}$ para temperaturas máximas médias às 15 e 18 UTC e mínimas médias às 09 UTC, mas na média a diferença ficou menor em torno de $1^{\circ}$ $\mathrm{C}$, enquanto outubro apresentou diferença entre as máximas médias e médias em torno de $4^{\circ} \mathrm{C}$ às 15 UTC e 18 UTC e mínimas médias em torno de $2^{\circ} \mathrm{C}$ às 09 UTC. Figura 7.

Foi observado que o modelo consegue simular bem o ciclo diário de temperatura, apresentando alguns dias de subestimação de temperatura em abril de 2019 e sistematicamente superestimando as máximas e subestimando as mínimas em outubro de 2019 Figuras 3 e 4 . Nos gráficos de dispersão dos dados verificou-se a diferença entre os meses de abril de 2019 e outubro de 2019, abril de 2019 comportou-se com maior dispersão ( $\mathrm{R} 2$ em torno de 0,35 a 0,55) e menor inclinação (coeficiente angular de 1,3 a 1,6), enquanto outubro de 2019 apresentou menor dispersão (R2 em torno de 0,9) e maior inclinação (coeficiente angular de 2,5 a 2,8) Figuras 5 e 6.

Analisando-se de forma geral, foram boas as previsões do RegCM4.7 para temperaturas máximas médias, médias e mínimas médias, nas quatro simulações para cada mês, e a média das simulações, em comparação com os dados de reanálise do ERA5, mostraram um bom desempenho do RegCM4.7. Para a previsão de temperatura máxima média o modelo tem melhor desempenho no mês de abril de 2019 superestimando em $2^{\circ} \mathrm{C}$, enquanto em outubro de 2019 superestima em $4^{\circ} \mathrm{C}$. $\mathrm{Na}$ previsão de temperatura média o modelo acerta bem nos dois meses, abril subestimando em $1^{\circ} \mathrm{C} \mathrm{e}$ melhor desempenho em outubro apresentando diferença mínima, quase coincidindo. Por fim, a temperatura mínima média do modelo tem comportamento semelhante nos dois meses de avaliação, subestimando em $2^{\circ} \mathrm{C}$ conforme observado na Figura 7. 
Figura 1 - Campos de temperatura média à superfície em graus Célsius: Eixo X longitudes de $041^{\circ} \mathrm{W}$ a $048^{\circ} \mathrm{W}$, eixo $\mathrm{Y}$ latitudes de $06^{\circ} \mathrm{S}$ a $01^{\circ} \mathrm{N}$. Acima Temperatura Média Observada em abril de 2019 (a) ERA5 (reanálise), abaixo simulações de temperaturas médias simuladas pelo modelo RegCM4.7 para o mês de abril de 2019: (b) inicializada em janeiro de 2019; (c) inicializada em fevereiro de 2019; (d) inicializada em março de 2019; e (e) inicializada em abril de 2019. (Autor).

a)
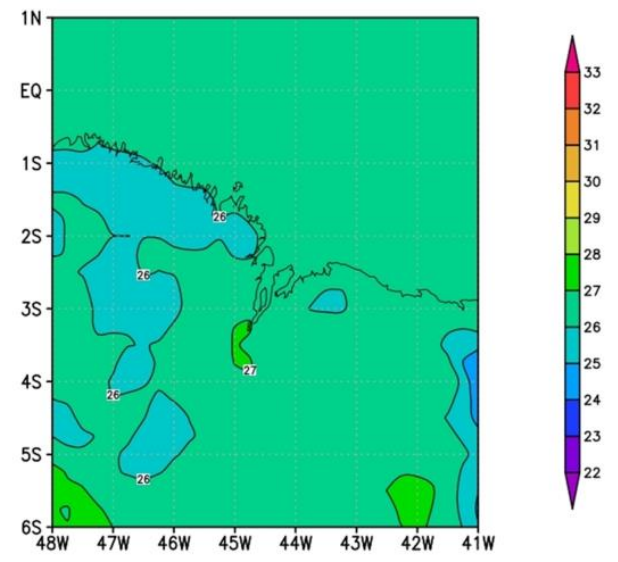

b)

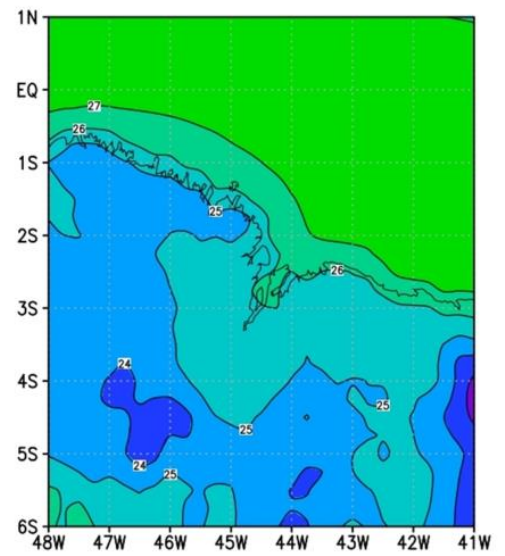

d)

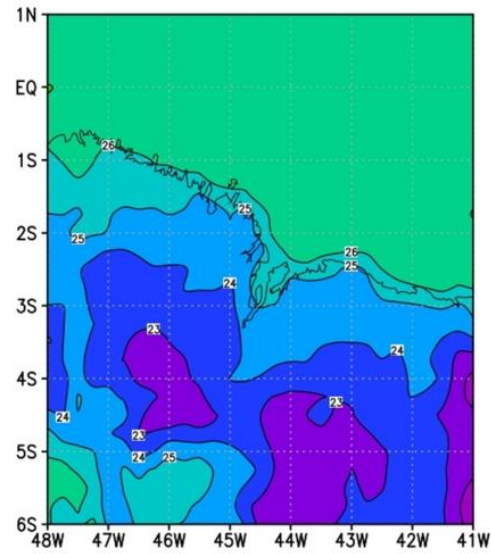

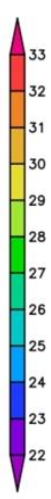

c)

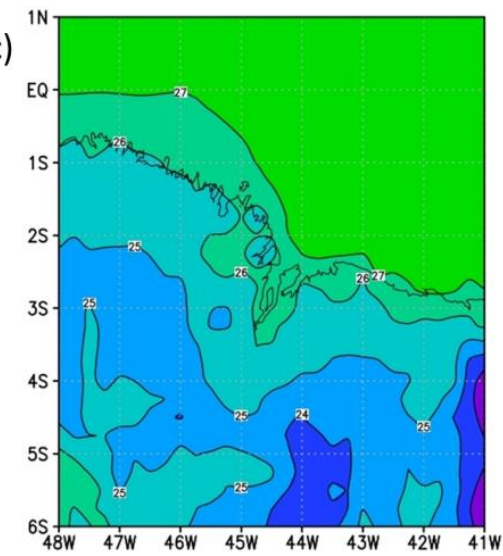

e)

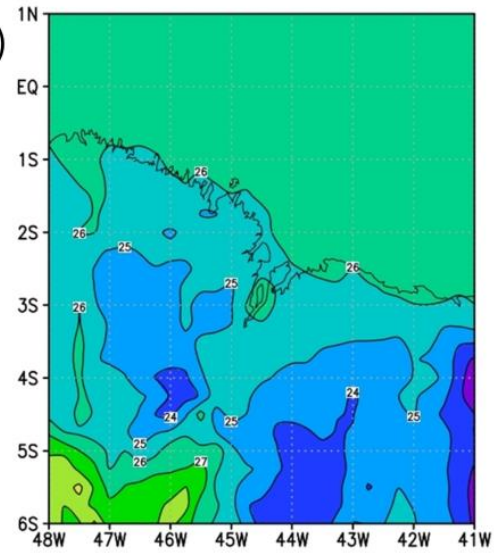


Figura 2 - Campos de temperatura média à superfície em graus Célsius: Eixo X longitudes de $041^{\circ} \mathrm{W}$ a $048^{\circ} \mathrm{W}$, eixo $\mathrm{Y}$ latitudes de $06^{\circ} \mathrm{S}$ a $01^{\circ} \mathrm{N}$. Acima Temperatura Média Observada em outubro de 2019 (a) ERA5 (reanálise), abaixo simulações de temperaturas médias simuladas pelo modelo RegCM4.7 para o mês de outubro de 2019: (b) inicializada em julho de 2019; (c) inicializada em agosto de 2019; (d) inicializada em setembro de 2019; e (e) inicializada em outubro de 2019. (Autor).

a)

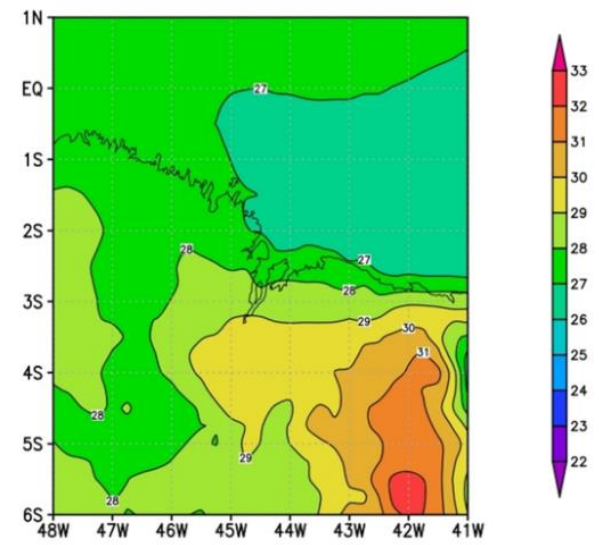

b)

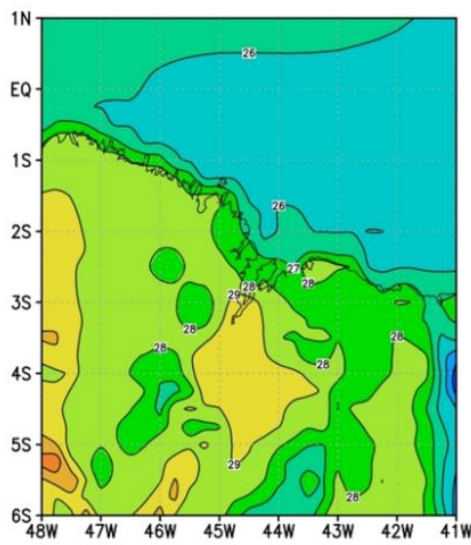

d)

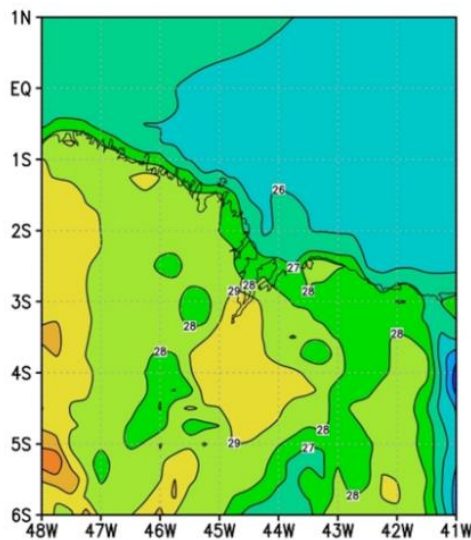

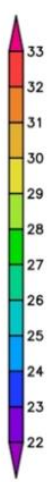

c)

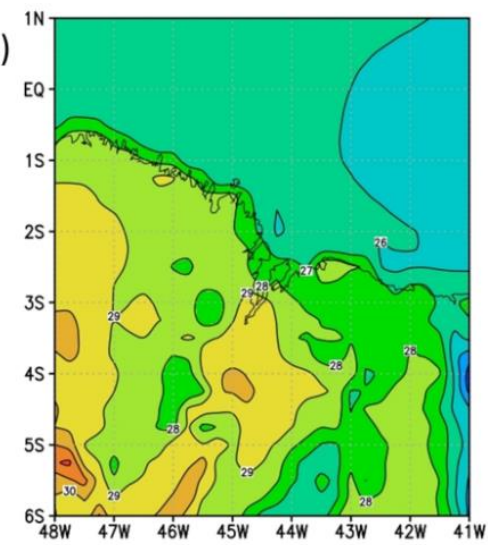

e)

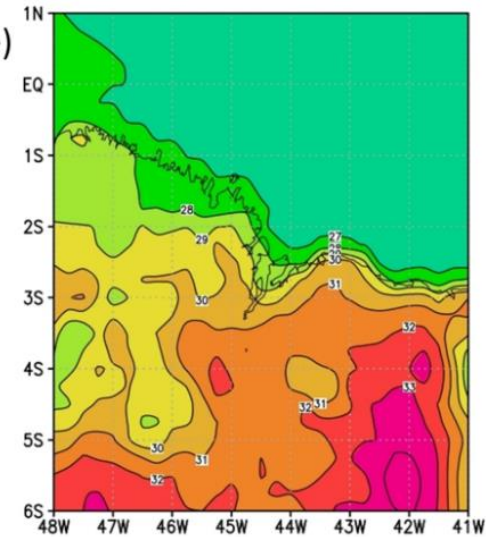




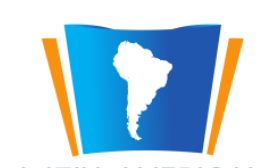

LATIN AMERICAN

Figura 3 - Temperaturas Previstas RegCM4.7 (laranja) e Temperaturas Observadas da Reanalise do ERA5 (azul), no Centro de Lançamento de Alcântara $\left(02^{\circ}, 15^{\prime} \mathrm{S}\right.$ e $\left.044^{\circ}, 30^{\prime} \mathrm{W}\right)$, para o mês de abril de 2019 com rodadas iniciadas em: a) janeiro de 2019; b) fevereiro de 2019; c) março de 2019; e d) abril de 2019. (Autor).
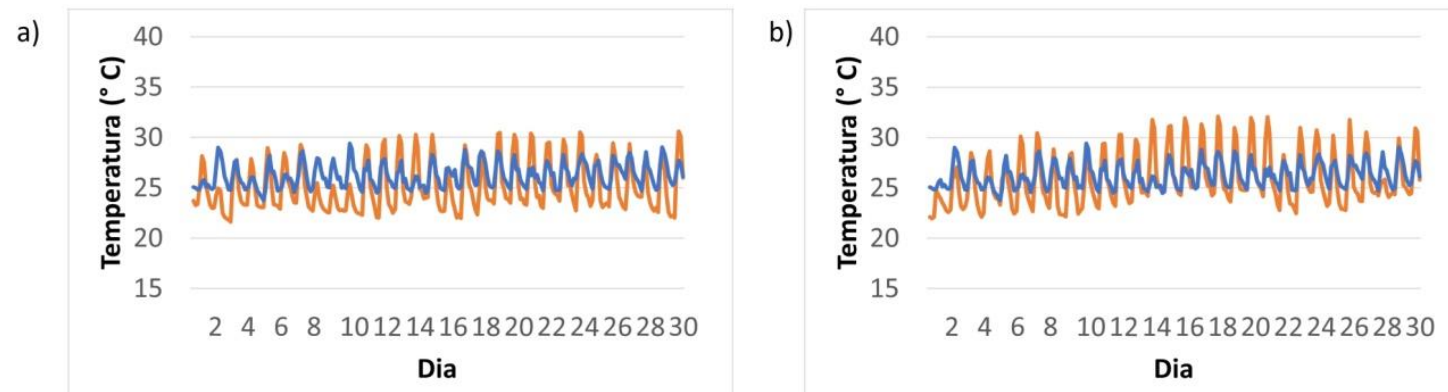

c)

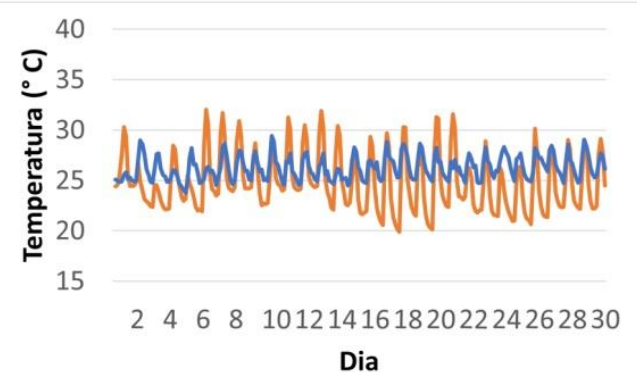

d)

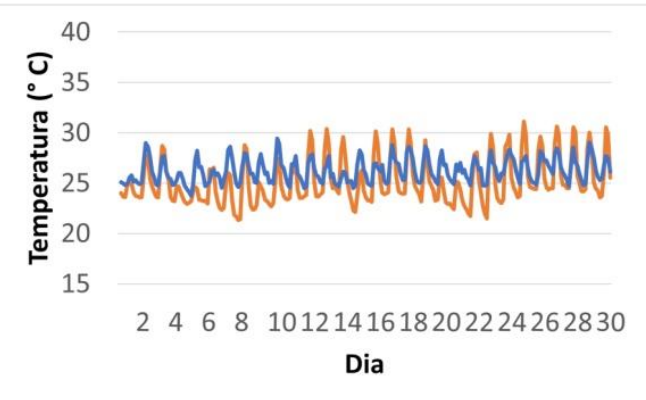

Figura 4 - Temperaturas Previstas RegCM4.7 (laranja) e Temperaturas Observadas da reanálise do ERA5 (azul), no Centro de Lançamento de Alcântara $\left(02^{\circ}, 15^{\circ} \mathrm{S}\right.$ e $\left.044^{\circ}, 30^{\prime} \mathrm{W}\right)$, para o mês de outubro de 2019 com rodadas iniciadas em: a) julho de 2019; b) agosto de 2019; c) setembro de 2019; e d) outubro de 2019. (Autor).

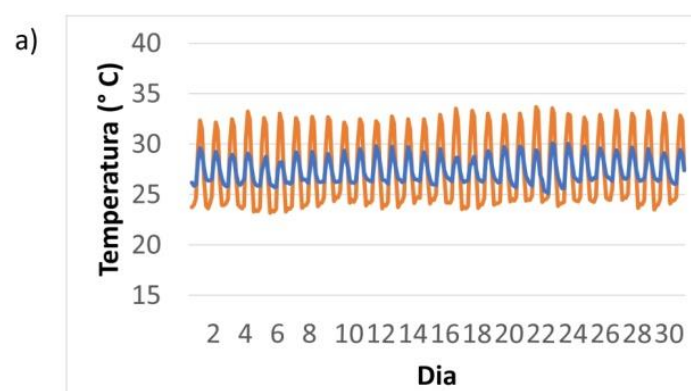

c)

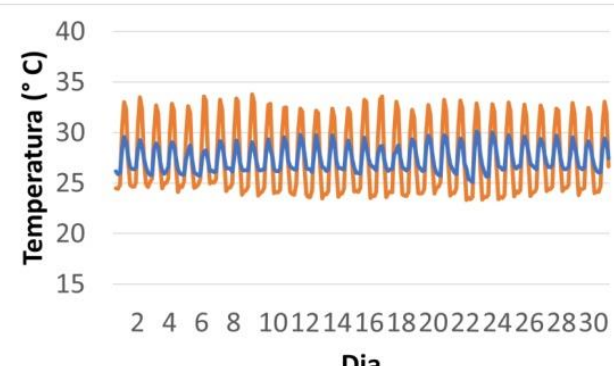

Dia b)

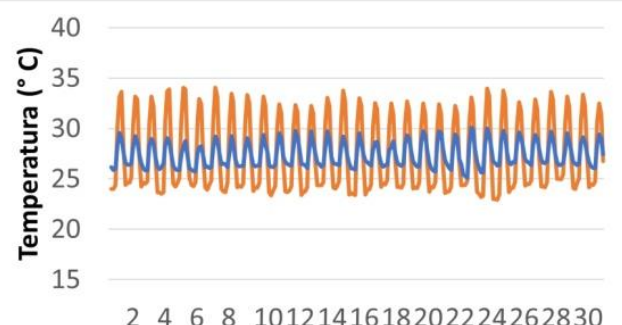

Dia

d)

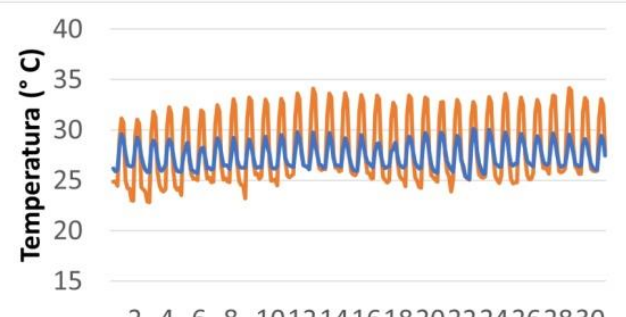

$2468 \quad 1012141618202224262830$

Dia 


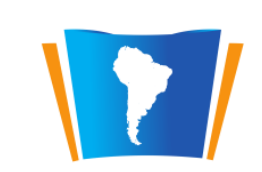

LATIN AMERICAN

Figura 5 - Gráficos de Dispersão das Temperaturas Observadas ERA5 (eixo X) e Temperaturas Previstas RegCM4.7 (eixo Y), no Centro de Lançamento de Alcântara $\left(02^{\circ}, 15^{\prime} \mathrm{S}\right.$ e $\left.044^{\circ}, 30^{\prime} \mathrm{W}\right)$, para o mês de abril de 2019 com rodadas iniciadas em: a) janeiro de 2019; b) fevereiro de 2019; c) março de 2019; e d) abril de 2019. (Autor).

a)

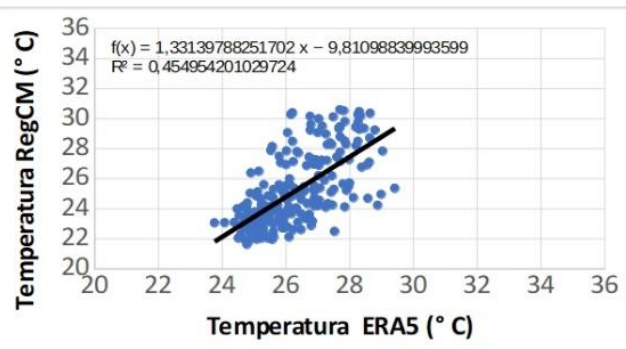

c)

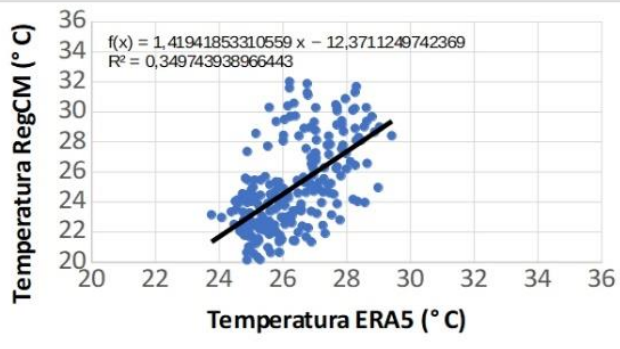

b)

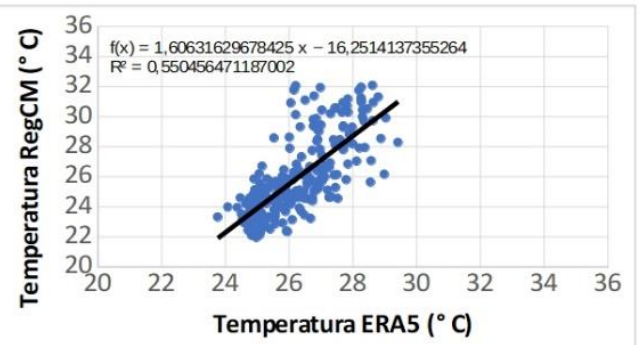

d)

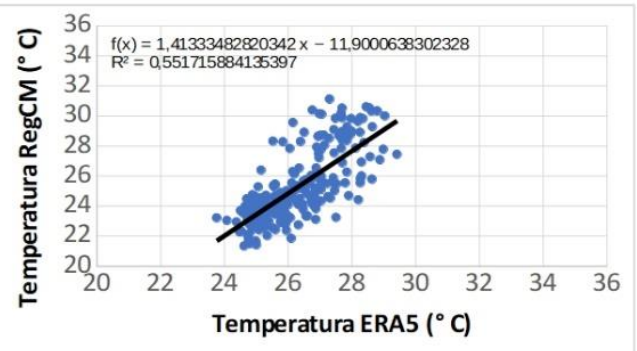

Figura 6 - Gráficos de Dispersão das Temperaturas Observadas ERA5 (eixo X) e Temperaturas Previstas RegCM4.7 (eixo Y), no Centro de Lançamento de Alcântara $\left(02^{\circ}, 15^{\prime}\right.$ 'S e $\left.044^{\circ}, 30^{\prime} \mathrm{W}\right)$, para o mês de abril de 2019 com rodadas iniciadas em: a) julho de 2019; b) agosto de 2019; c) setembro de 2019; e d) outubro de 2019. (Autor).

a)

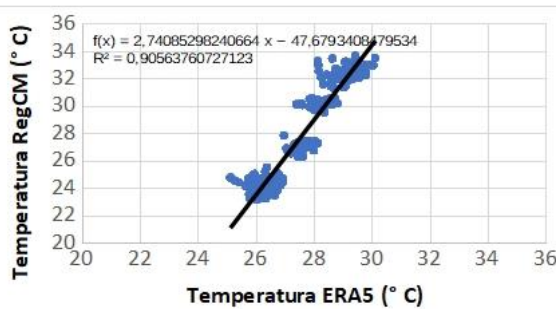

c)

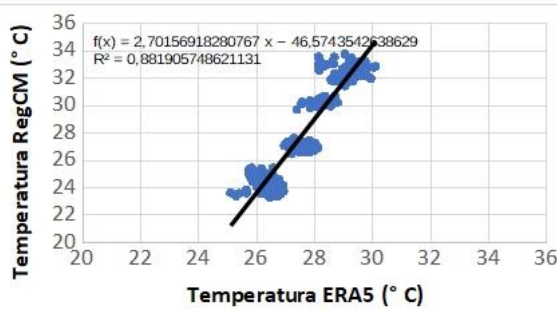

b)

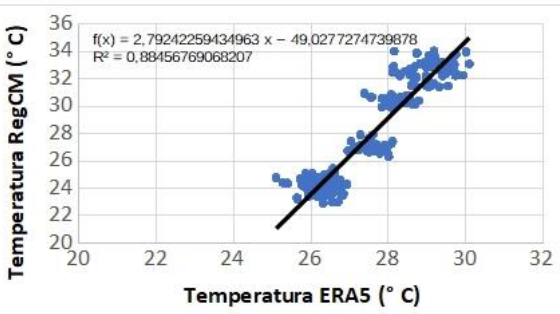

d)

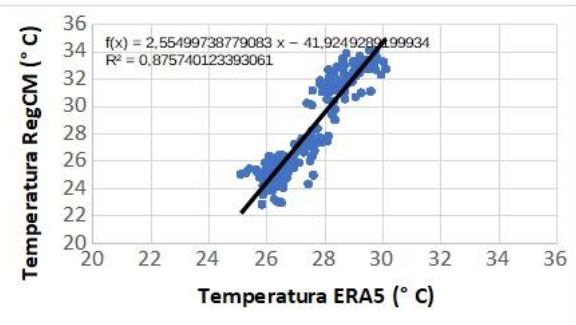


Figura 7 - Comparação de Temperatura Máxima Média Observada ERA5 (azul) e Prevista RegCM (laranja), no Centro de Lançamento de Alcântara (02, $15^{\prime} \mathrm{S}$ e $\left.044^{\circ}, 30^{\prime} \mathrm{W}\right)$, para os meses de (a) abril de 2019 e (b) outubro de 2019;

Comparação de Temperatura Média Observada ERA5 (azul) e Prevista RegCM (laranja), no Centro de Lançamento de Alcântara $\left(02^{\circ}, 15^{\prime} \mathrm{S}\right.$ e $\left.044^{\circ}, 30^{\prime} \mathrm{W}\right)$, para os meses de (c) abril de 2019 e (d) outubro de 2019; e Comparação de Temperatura Mínima Média Observada ERA5 (azul) e Prevista RegCM4.7 (laranja), no Centro de Lançamento de Alcântara $\left(02^{\circ}, 15^{\prime}\right.$ S e $044^{\circ}, 30^{\prime} \mathrm{W}$ ), para os meses de (e) abril de 20190e (f) outubro de 2019. (Autor).

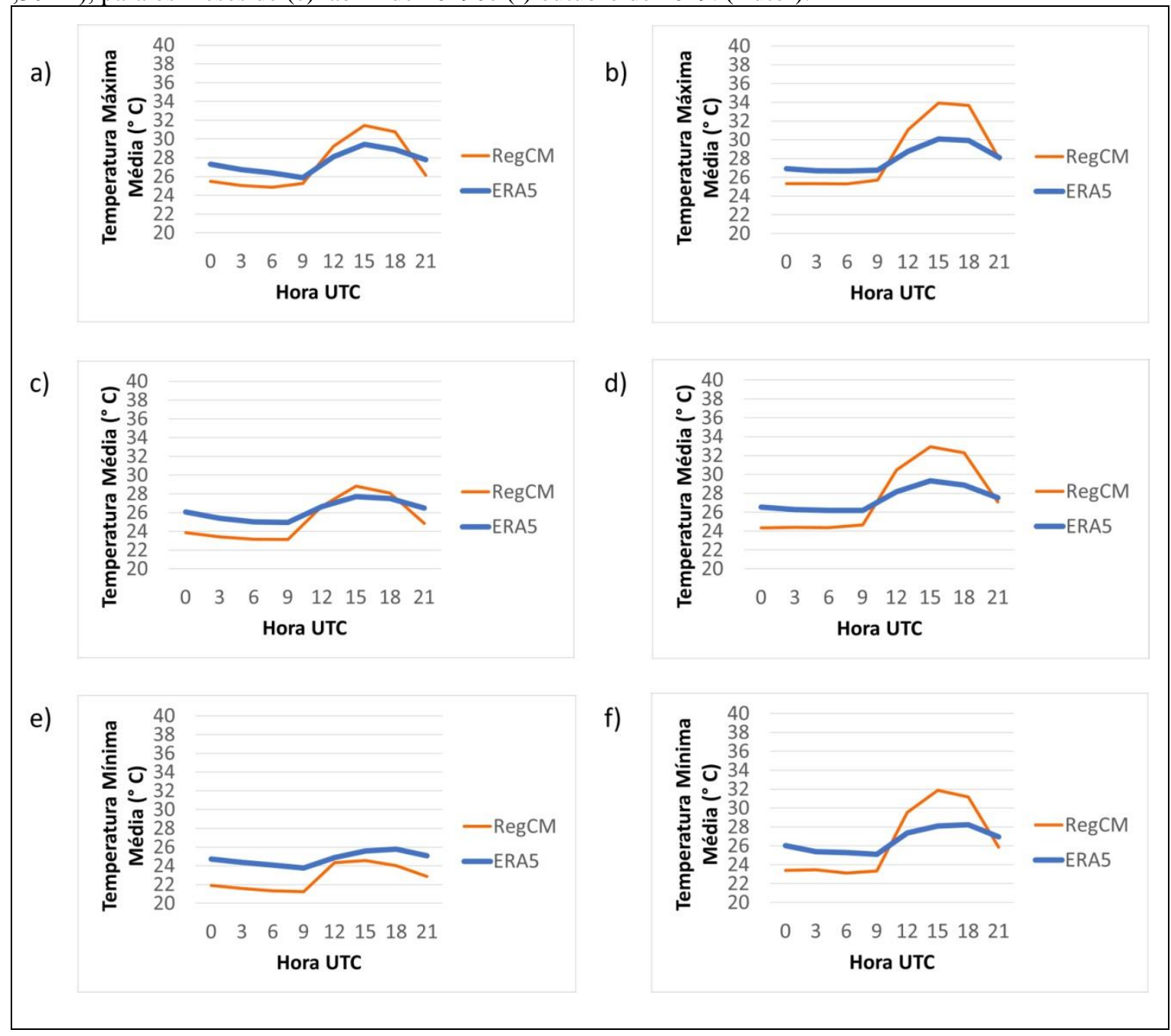

\section{CONSIDERAÇÕES FINAIS.}

O Sol é o principal responsável pelas variações de temperatura em qualquer região da superfície do planeta Terra. No entanto as superfícies com terra ou oceanos também interferem nas temperaturas das regiões do globo terrestre. As avaliações das previsões de temperatura à superfície para a região do CLA nos meses de abril de 2019 e outubro de 2019 indicaram um bom desempenho geral para o modelo RegCM4.7 na previsão de temperaturas médias, o que contribuirá no conhecimento da utilização dessas previsões em apoio ao planejamento de operações de lançamento, visto que, tanto para meses chuvosos como secos, o desempenho geral do modelo para previsão de temperaturas médias é muito bom. Contudo, sabendo-se da tendência do RegCM4.7 em superestimar temperaturas máximas médias e subestimar temperaturas mínimas médias, ainda assim, com as devidas correções, poderá ser utilizado 
com eficácia para a previsão intrasazonal de temperatura à superfície em apoio ao planejamento de operações de lançamento no CLA.

\section{AGRADECIMENTOS}

Ao Instituto Tecnológico de Aeronáutica - ITA, PG-CTE-F pelo apoio nas orientações; ao Instituto de Aeronáutica e Espaço - IAE, ACA pela disponibilização de dados de simulações do RegCM4.7 e orientações. 


\section{REFERÊNCIAS BIBLIOGRÁFICAS}

[1] LIMA, P. C. M.; MARTIN, I. M. Variações do Clima Espacial: Possíveis implicações Climáticas nas regiões equatorial e sul do Atlântico. Tese de Mestrado defendida em 23 de junho de 2021 no ITA no PG-CTE-F e aprovada -.

[2] CARUZO M.; BELDERRAINET M. C. N.; FISCH G. F. Mapeamento parcial da meteorologia nas operações de lançamento de foguetes utilizando um Método de Estruturação de Problemas, XVI Simpósio de Aplicações Operacionais em Áreas de Defesa, São José dos Campos, p. 154, 2014.

[3] CORREA, C. S.; CAMILlO, G. L.; COUTO, V. M.; FISCH, G.; CORREA, F. N.; HARTER, F. Climate Forecasts at the Centro de Lançamento de Alcântara Using the Climate Model RegCM4. Journal of Aerospace Technology and Management, São José dos Campos, Vol.9, $\mathrm{N}^{\circ}$ 1, p. 18-28, Jan.-Mar. 2017.

[4] CHOU, S. C. NOBRE, P. Avaliação de Modelos Globais e Regionais Climáticos. Instituto Nacional de Pesquisas Espaciais. São José dos Campos, p. 280-331, 2011.

[5] RUMMUKAINEN, M. State-of-the-art with regional climate models, WIREs Climate Change.Vol. $1, \quad$ p.82, Jan-Feb. $2010 . \quad$ Disponível em: http://danida.vnu.edu.vn/cpis/files/Papers_on_CC/CC/State-of-theart\%20with\%20regional\%20climate\%20models.pdf. Acesso em: 14 out. 2019.

[6] DICKINSON, R. E.; Errico R. M.; Giorgi F.; et al.A regional climate model for the western United States. Clim. Change 15: p. 383-422, 1989.

[7] GIORGI, F. \& BATES G. T. The climatological skill of a regional model over colmplex terrain. Mon. Wheather Ver. 117: p. 2325-2347, 1989.

[8] GIORGI, F. Simulation of regional climate using a limited área model a general nested in circulation model.J. Clim. 3: p. 941-963, 1990.

[9] AMBRIZZI, T.; REBOITA, M. S.; ROCHA, R. P.; LLOPART M. The State-of-the-art and Fundamental Aspects of Regional Climate Modeling in South America. Annals of The New York Academy of Sciences, New York, p. 1-23, 2018.

[10] DA ROCHA, R. P.; CUADRA, S. V.; REBOITA, M. S.; KRÜGER, L. F.; AMBRIZZI, T.; KRISCHE, N. Effects of RegCM3 parameterizations on simulated rainy season over South America. Climate Research, (2012) v. 52, p. 253-265.

[11] REBOITA, M. S.; FERNANDEZ, J. P. R.; LLOPART, M. P.; Da ROCHA, R. P.; PAMPUCH, L. A.; CRUZ, F. T. M. S.; Assessment of RegCM4.3 over the CORDEX South America domain: sensitivity analysis for physical parameterization schemes. Clim. Res. (2014), 60:215-234. https://doi.org/10.3354/cr01239

[12] REBOITA, M. S.; DIAS C. G.; DUTRA L. M. M.; ROCHA, R. P.; LLOPART M. Previsão Climática Sazonal para o Brasil Obtida Através de Modelos Climáticos Globais e Regional. Revista Brasileira de Meteorologia, São José dos Campos, v. 33, n. 2, p. 207-224, 2018. 
[13] CORREA, C. S.; HARTER, F. P.; CAMILLO, G. L. Intraseasonal Ensemble Forecasting for the Brazilian Northeastern. Ciência e Natura, Santa Maria, v.41, e10, p.01-08, jul. 2019.

[14] PEREIRA, E. I.; MIRANDA, I.; FISCH, G. F.; MACHADO, L. A. T.; ALVES, M. A. S. Atlas Climatológico do Centro de Lançamento de Alcântara. São José dos Campos: Centro Técnico Aeroespacial, 2002. Relatório de desenvolvimento, ACA/RT-01/01, GDO-000000/B0047.

[15] ICTP. Site do International Center for Theoretical Phisics, 2021. RegCM4-A Regional Climate Model system. Disponível em: https://www.ictp.it/research/esp/models/regcm4.aspx. Acesso em: 20 jan. 2021.

[16] NCEP. Site do National Centers for Enviromental Prediction, 2021. Climate Forecast System. Disponível em: https://cfs.ncep.noaa.gov/cfsv2/downloads.html

Acesso em: 20 jan. 2021.

[17] ECMWF. Site do European Centre for Medium-Range Weather Forecasts, 2021. ERA5-Land hourly data from 1981 to present. Disponível em: https://cds.climate.copernicus.eu/cdsapp\#!/dataset/reanalysis-era5-land?tab=overview. Acesso em: 30 jan. 2021.

[18] SANTOS, J. G. M. Introdução ao Climate Data Operator (CDO). Instituto Nacional de Pesquisas Espaciais, São José dos Campos, 2014.

[19] SOUZA, E. B. GraDS - Grid Analysis and Display Sistem Fundamentos e Programação Básica, Universidade Federal do Pará, Belém, fevereiro, 2004. 